\title{
ON SOME INEQUALITIES FOR GENERALIZED BETA FUNCTION
}

\author{
LJ. Dedić, M. MATIĆ AND J. PEČARIĆ
}

Abstract. Some integral representations and inequalities for generalized beta function are given.

Mathematics subject classification (1991): 26D10, 26D15, 26D99.

Key words and phrases: Inequalities, generalized beta function.

\section{REFERENCES}

[1] DRAgOMiR, R. P. AgaRWAL AND N. S. BARNETT, Inequalities for beta and gamma functions via some classical and new integral inequalities, RGMIA, Vol. 2, No. 3.

[2] LJ. Dedić, On Haar measure on SL(n,R), Publ. Instit. Math., Nouvelle serie tome 47 (61), 1990, 56-60.

[3] H. FEDERER, Geometric measure theory, Grundlagen der math. Wissenschaften, Band 153, 1969. 